\title{
Intra-Amniotic Infection and Sterile Intra-Amniotic Inflammation in Cervical Insufficiency with Prolapsed Fetal Membranes: Clinical Implications
}

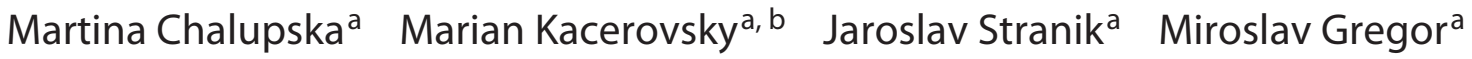 \\ Jan Malyc Bo Jacobsson d, e, f Ivana Musilova ${ }^{a}$ \\ aDepartment of Obstetrics and Gynecology, University Hospital Hradec Kralove, Hradec Kralove, Czech Republic; \\ ${ }^{\mathrm{b}}$ Biomedical Research Center, University Hospital Hradec Kralove, Hradec Kralove, Czech Republic; 'Department \\ of Pediatrics, University Hospital Hradec Kralove, Hradec Kralove, Czech Republic; ${ }^{\mathrm{d} D e p a r t m e n t}$ of Obstetrics and \\ Gynecology, Institute of Clinical Science, Sahlgrenska Academy, University of Gothenburg, Gothenburg, Sweden; \\ eRegion Västra Götaland, Department of Obstetrics and Gynecology, Sahlgrenska University Hospital, Gothenburg, \\ Sweden; fDepartment of Genetics and Bioinformatics, Domain of Health Data and Digitalisation, Institute of Public \\ Health, Oslo, Norway
}

\section{Keywords}

Amniocentesis · Cerclage · Emergency cerclage ·

Microorganism $\cdot$ Mycoplasma $\cdot$ Interleukin- $6 \cdot$ MIAC .

Microbial invasion of the amniotic cavity $\cdot$ Nonelective cerclage $\cdot$ Physical exam-indicated cerclage $\cdot$ Preterm delivery $\cdot$ Rescue cerclage $\cdot$ Second trimester abortion . Second trimester loss · Sterile inflammation · Ureaplasma

\footnotetext{
Abstract

Introduction: The aim of this study was to identify the rates of 2 phenotypes of intra-amniotic inflammation: intra-amniotic infection (with microbial invasion of the amniotic cavity $[M I A C]$ ) and sterile intra-amniotic inflammation (without MIAC), and their outcomes, among women with cervical insufficiency with prolapsed fetal membranes. Methods of Study: This is a retrospective study of women admitted to the Department of Obstetrics and Gynecology, University Hospital Hradec Kralove between January 2014 and May 2020. Transabdominal amniocentesis to evaluate intra-amniotic inflammation (amniotic fluid interleukin-6) and MIAC (culturing and molecular biology methods) was performed as part of standard clinical management. Results: In total, 37
}

women with cervical insufficiency and prolapsed fetal membranes were included; $11 \%(4 / 37)$ and $43 \%(16 / 37)$ of them had intra-amniotic infection and sterile intra-amniotic inflammation, respectively. In women with intra-amniotic infection and sterile intra-amniotic inflammation, we noted shorter intervals between admission and delivery (both $p<$ $0.0001)$, and lower gestational age at delivery $(p<0.0001$ and $p=0.004$ ) and percentiles of birth/abortion weight ( $p=$ 0.03 and $p=0.009$, respectively) than in those without intraamniotic inflammation. Conclusions: Both phenotypes of intra-amniotic inflammation, with sterile intra-amniotic inflammation being more frequent, are associated with worse outcomes in pregnancies with cervical insufficiency with prolapsed fetal membranes.

(c) 2020 The Author(s) Published by S. Karger AG, Basel

\section{Introduction}

Cervical insufficiency is traditionally defined as a dilatation of the cervix in the second trimester with absence of uterine contractions and/or vaginal bleeding [1-3]. The most pronounced form of cervical insufficiency is as-
(C) 2020 The Author(s)

Published by S. Karger AG, Basel

This is an Open Access article licensed under the Creative Commons Attribution-NonCommercial-4.0 International License (CC BY-NC) (http://www.karger.com/Services/OpenAccessLicense), applicable to the online version of the article only. Usage and distribution for commercial purposes requires written permission.
Marian Kacerovsky

Biomedical Research Center

University Hospital Hradec Kralove

Sokolska 581, CZ-500 05 Hradec Kralove (Czechia)

kacermar@fnhk.cz 
sociated with the prolapse of fetal membranes at or beyond the external cervical os [4]. This condition represents a rare but serious pregnancy complication owing to associations with poor pregnancy outcomes such as abortion or preterm delivery $[1,2,5,6]$.

There is a solid body of evidence demonstrating that cervical insufficiency with prolapsed fetal membranes is an indication for cervical cerclage $[1-3,7,8]$. Apart from advanced cervical dilatation $[8,9]$, the success of treatment with cervical cerclage in terms of prolongation of pregnancy mainly depends on the absence of intra-amniotic complications such as intra-amniotic inflammation and/or microbial invasion of the amniotic cavity (MIAC) $[4,10-13]$. These complications may occur in more than half the pregnancies with cervical insufficiency with prolapsed fetal membranes [4, 10, 12, 14-17].

A clinical dilemma still remains regarding the treatment of cases with intra-amniotic inflammation and/or MIAC since cervical cerclage placement is not recommended by certain authors and standard antibiotic treatment does not usually work [10-12, 17-19]. However, Oh et al. [20] have recently shown that treatment with ceftriaxone, clarithromycin, and metronidazole may resolve intra-amniotic inflammation in women with cervical insufficiency and prolapsed fetal membranes. Therefore, the use of amniocentesis to characterize an intra-amniotic environment might be an important step in the decision-making process for a pregnancy with cervical insufficiency with prolapsed fetal membranes $[4,10,11,13$, 17-19].

Intra-amniotic inflammation has 2 different phenotypes based on the triggering stimulus: (i) intra-amniotic infection, when MIAC is present and (ii) sterile intra-amniotic inflammation, when MIAC is absent [21-23]. Intra-amniotic infection and sterile intra-amniotic inflammation complicate approximately 2 and $10 \%$ of pregnancies, respectively, with a sonographic short cervix $[23,24]$. Nevertheless, there is a gap in our knowledge of the rate and proportion of both types of intra-amniotic inflammation and their impacts on the outcomes of pregnancies with cervical insufficiency with prolapsed fetal membranes.

Therefore, the first aim of this study was to identify the rates of intra-amniotic infection and sterile intra-amniotic inflammation among pregnancies with cervical insufficiency with prolapsed fetal membranes. The study further aimed to assess the following: (1) the interval from admission to delivery and gestation age at delivery; (2) the effect of antibiotic treatment; (3) the percentiles of birth/ aborted weight of the newborns/aborted fetuses; and (4) short-term neonatal morbidity with respect to the presence of intra-amniotic infection and sterile intra-amniotic inflammation.

\section{Material and Methods}

This was a retrospective study of the patients admitted to the Department of Obstetrics and Gynecology, University Hospital Hradec Kralove between August 2014 and May 2020 who met the following criteria: (1) singleton pregnancy; (2) gestational age between $18+0$ or $26+6$ weeks; (3) painless cervical dilatation $\geq 1 \mathrm{~cm}$ with visible intact fetal membranes prolapsed at or beyond the external cervical os, confirmed by sterile speculum examination; (4) absence of uterine contractions; (5) absence of chromosomal and/ or structural fetal abnormalities.

\section{Management of Cervical Insufficiency with Prolapsed Fetal} Membranes

Gestational age was established by first-trimester fetal biometry. Cervical dilatation and prolapsed fetal membranes were determined by speculum examination at the time of admission. The performance of transabdominal amniocentesis to assess the intraamniotic environment (interleukin [IL]-6 concentrations and MIAC), part of standard management of such pregnancy pathologies at our department, was offered to all women admitted with cervical insufficiency with prolapsed fetal membranes.

All women with gestational age at admission $\geq 24+0$ weeks received a course of corticosteroids (betamethasone $14 \mathrm{mg}$ intramuscularly, $24 \mathrm{~h}$ apart). A decision on the treatment of cervical insufficiency with prolapsed fetal membranes was made once the result of amniotic fluid IL-6 was available. Women without intra-amniotic inflammation did not receive any antibiotics and were treated with a cerclage performed on the following working day. All cerclages placed were of the McDonald type. Women with intra-amniotic inflammation received intravenous antibiotic therapy. They were administered clarithromycin, $500 \mathrm{mg}$ every $12 \mathrm{~h}$, for 7 days, unless an abortion or delivery occurred. Once the results from culturing and/or PCR were known, the attending clinician charted individualized treatments to determine the optimal antibiotic therapy. A follow-up amniocentesis was offered to all women with intraamniotic inflammation when the antibiotic therapy ended. None of the women received tocolytic therapy upon admission.

\section{Amniotic Fluid Sampling}

Ultrasound-guided transabdominal amniocentesis was performed at the time of admission, before treatment initiation. Approximately $5 \mathrm{~mL}$ of amniotic fluid was aspirated and divided into 4 tubes. The first tube was used for assessment of IL-6 levels in amniotic fluid and the remaining tubes were transported to the laboratory for (i) aerobic and anaerobic cultures of amniotic fluid; (ii) PCR detection of Ureaplasma spp., Mycoplasma hominis, and Chlamydia trachomatis; and (iii) PCR detection and sequencing of $16 \mathrm{~S}$ rRNA gene.

\section{Assessment of IL-6 Concentrations in Amniotic Fluid}

Concentrations of IL- 6 in amniotic fluid samples obtained between August 2014 and November 2018 were assessed using Milenia QuickLine IL-6 lateral flow immunoassay and a Milenia POCScan Reader (Milenia Biotec, GmbH, Giessen, Germany). The 
measurement range was $50-10,000 \mathrm{pg} / \mathrm{mL}$. [25]. The concentration of IL-6 in amniotic fluid samples obtained between December 2018 and May 2020 were evaluated using an automated electrochemiluminescence immunoassay method with immuno-analyzer Cobas e602, which is part of the Cobas 8000 platform (Roche Diagnostics, Basel, Switzerland). The measurement range was 1.5$5,000 \mathrm{pg} / \mathrm{mL}$, which could be extended to $50,000 \mathrm{pg} / \mathrm{mL}$ with a 10 -fold dilution of the sample [26].

\section{Aerobic and Anaerobic Cultures of Amniotic Fluid}

Amniotic fluid samples were cultured in Columbia agar with sheep's blood, Gardnerella vaginalis selective medium, MacConkey agar, Neisseria selective medium (modified Thayer-Martin medium), Sabouraud agar, and Schaedler anaerobe agar. The plates were cultured for 6 days and checked daily. Species were identified by matrix-assisted laser desorption/ionization time-offlight mass spectrometry using MALDI Biotyper software (Bruker Daltonics, Billerica, MA, USA).

PCR Detection of Ureaplasma Species, M. hominis, and C. trachomatis in Amniotic Fluid

DNA was isolated from the amniotic fluid using a QIAamp DNA Mini Kit, as per manufacturer instructions (using the protocol for isolating bacterial DNA from biological fluids). RT-PCR was performed using a Rotor-Gene 6000 instrument and a commercial AmpliSens ${ }^{\circledR}$ C. trachomatis/Ureaplasma/M. hominis-FRT Kit (Federal State Institution of Science, Central Research Institute of Epidemiology, Moscow, Russia) to detect DNA from Ureaplasma species, M. hominis, and C. trachomatis in the same PCR tube. As a control, we amplified $\beta$-actin, a housekeeping gene, to exclude the presence of PCR inhibitors.

PCR Detection and Sequencing of $16 S$ rRNA Gene

Bacterial DNA was identified by PCR targeting 16S rRNA using the following primers: $5^{\prime}$-CCAGACTCCTACGGGAGGCAG- ${ }^{\prime}$ (V3 region) and 5'-ACATTTCACAACACGAGC-GACGA-3' (V6 region) [27]. Each reaction contained $3 \mu \mathrm{L}$ target DNA, $500 \mathrm{nM}$ forward and reverse primers, and Q5 High-Fidelity DNA Polymerase (New England Biolabs, Ipswich, MA, USA) in a total volume of 25 $\mu \mathrm{L}$. Amplification was performed using a 2720 Thermal Cycler (Applied Biosystems, Foster City, CA, USA). The products were visualized on an agarose gel. Positive reactions yielded $950 \mathrm{bp}$ products that were subsequently analyzed by sequencing. The 16S PCR products were purified and sequenced by PCR using the above primers and BigDye Terminator v3.1 Cycle Sequencing Kit (Thermo Fisher Scientific, Waltham, MA, USA). The bacteria were then typed using sequences obtained from BLAST ${ }^{\circledR}$ and SepsiTest ${ }^{\mathrm{TM}}$ BLAST.

\section{Clinical Diagnosis}

Intra-amniotic inflammation was defined as concentration of IL- 6 in amniotic fluid $\geq 745 \mathrm{pg} / \mathrm{mL}$, when IL- 6 was measured using a lateral flow immunoassay point-of-care test $[28,29]$, or $\geq 3,000$ $\mathrm{pg} / \mathrm{mL}$, when IL- 6 was measured using the automated electrochemiluminescence immunoassay method [26]. MIAC was defined as the presence of microorganisms in amniotic fluid detected by culture and/or microbial nucleic acids in amniotic fluid. Intra-amniotic infection was defined as the presence of both intra-amniotic inflammation and MIAC. Sterile intra-amniotic inflammation was defined as the presence of intra-amniotic inflammation without the concomitant presence of MIAC.

\section{Diagnosis of Histologic Chorioamnionitis and Funisitis}

The degree of polymorphonuclear leukocyte infiltration was assessed separately in the free membranes (amnion and choriodecidua), chorionic plate, and umbilical cord. The diagnosis of histologic chorioamnionitis was based on the presence of inflammatory changes in the choriodecidua (grades 3-4), chorionic plate (grades 3-4), umbilical cord (grades 1-4), and/or amnion (grades 1-4). The diagnosis of funisitis was based on the presence of inflammatory changes in the umbilical cord (grades 1-4) [30].

\section{Birth/Abortion Weight Percentiles}

The Olsen 2010 growth calculator was used to calculate the percentiles for birth/abortion weights of newborns [31, 32]. Small for gestational age was defined as a birth/abortion weight below the 10th percentile for gestational age.

\section{Definitions of Selected Aspects of Short-Term Neonatal}

Morbidity

Maternal and perinatal medical records were reviewed by 5 investigators (M.C., J.S., I.M., J.M., and M.K.). Data regarding shortterm neonatal morbidity were reviewed for all newborns. "Compound neonatal morbidity" was defined as follows: the need for intubation, and/or respiratory distress syndrome (it is defined by the presence of 2 or more of the following criteria: evidence of respiratory compromise, persistent oxygen requirement for $>24 \mathrm{~h}$, administration of exogenous surfactant, and radiographic evidence of hyaline membrane disease), and/or bronchopulmonary dysplasia (defined as infant oxygen requirement or ventilator support at 36 weeks post-menstrual age), and/or pneumonia (diagnosed by abnormal findings on chest $\mathrm{X}$ rays), and/or retinopathy of prematurity (identified using retinoscopy), and/or intraventricular hemorrhage (diagnosed by cranial ultrasound examinations as described by Papile et al. [33]), and/or necrotizing enterocolitis (defined as radiological findings of either intramural gas or free intra-abdominal gas), and/or intestinal perforation, and/or early(during the first $72 \mathrm{~h}$ of life) or late-onset (between the ages of 4 and 120 days) sepsis (either proven by bacterial culture or clinically highly suspected sepsis), and/or death of the infants before hospital discharge.

\section{Statistical Analyses}

The demographic characteristics were compared using a nonparametric Mann-Whitney $U$ test for continuous variables and Fisher's exact test for categorical variables and presented as a median (interquartile range [IQR]) and numbers (\%), respectively. The normality of the data was tested using the Anderson-Darling test. Kaplan-Meier survival curves were constructed and log-rank (Mantel-Cox) test was used to compare survival distributions among women with intra-amniotic infection, with sterile intraamniotic inflammation, and without intra-amniotic inflammation. The percentiles of birth/abortion weight were compared using the nonparametric Mann-Whitney U test. Spearman partial correlation was used to adjust results for confounders. Differences were considered significant at $p<0.05$. All $p$ values were obtained from two-tailed tests, and all statistical analyses were performed using GraphPad Prism version 6.0h software for Mac OS X (GraphPad Inc., San Diego, CA, USA) and Statistical Package for Social Sciences (SPSS) version 19.0 for Mac OS X (SPSS Inc., Chicago, IL, USA).
60

Fetal Diagn Ther 2021;48:58-69

DOI: $10.1159 / 000512102$
Chalupska/Kacerovsky/Stranik/Gregor/

Maly/Jacobsson/Musilova 


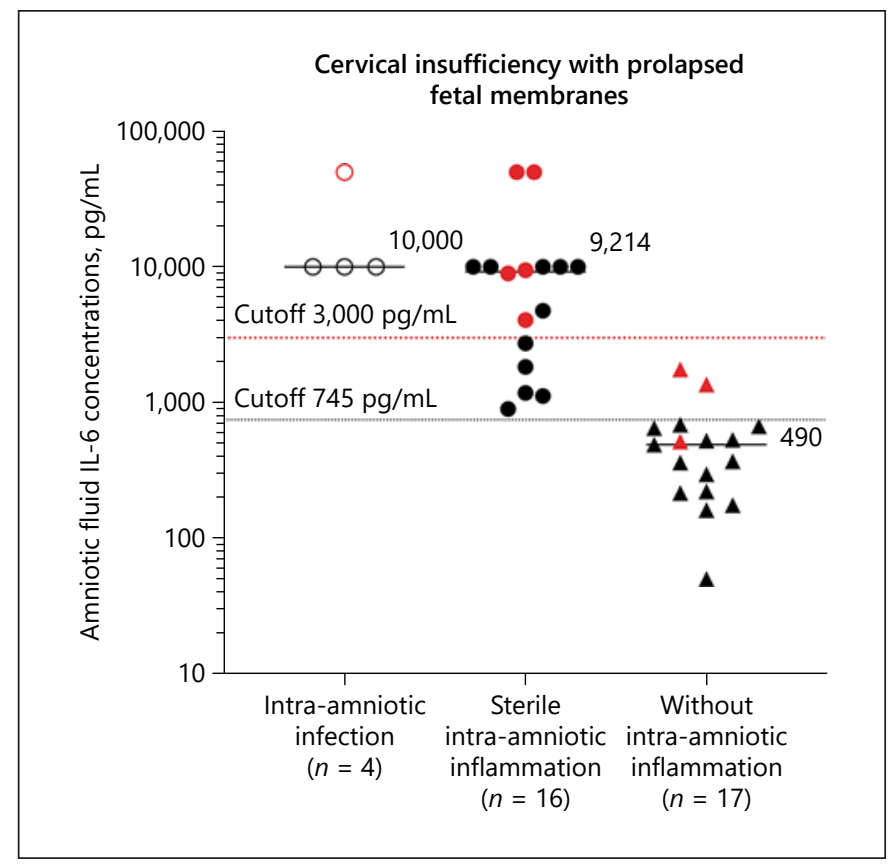

Fig. 1. Amniotic fluid IL-6 concentrations in women with cervical insufficiency with prolapsed fetal membranes: (i) with intra-amniotic infection; (ii) with sterile intra-amniotic inflammation; and (iii) without intra-amniotic inflammation. Amniotic fluid concentrations measured using lateral flow immunoassay method are marked in black and those measured using electrochemiluminescence immunoassay method are marked in red. The black dotted line indicates the cutoff value for intra-amniotic inflammation when IL-6 was measured using lateral flow immunoassay and red indicates the cutoff value for intra-amniotic inflammation when IL- 6 was measured by the electrochemiluminescence immunoassay method. IL, interlukin.

\section{Results}

In total, 37 women with singleton pregnancy complicated by cervical insufficiency with prolapsed fetal membranes were included. Among them, 54\% (20/37) had intra-amniotic inflammation and were treated with intravenous antibiotics. The remaining 46\% (17/37) did not experience intra-amniotic inflammation and underwent cerclage. MIAC was found in $11 \%(4 / 37)$ of the women and only in those with intra-amniotic inflammation. Demographic and clinical data of the women with cervical insufficiency with prolapsed membranes have been presented in Table 1.

\section{Intra-Amniotic Infection and Sterile Intra-Amniotic Inflammation}

Among those with intra-amniotic inflammation, 20\% $(4 / 20)$ and $80 \%(16 / 20)$ had intra-amniotic infection and

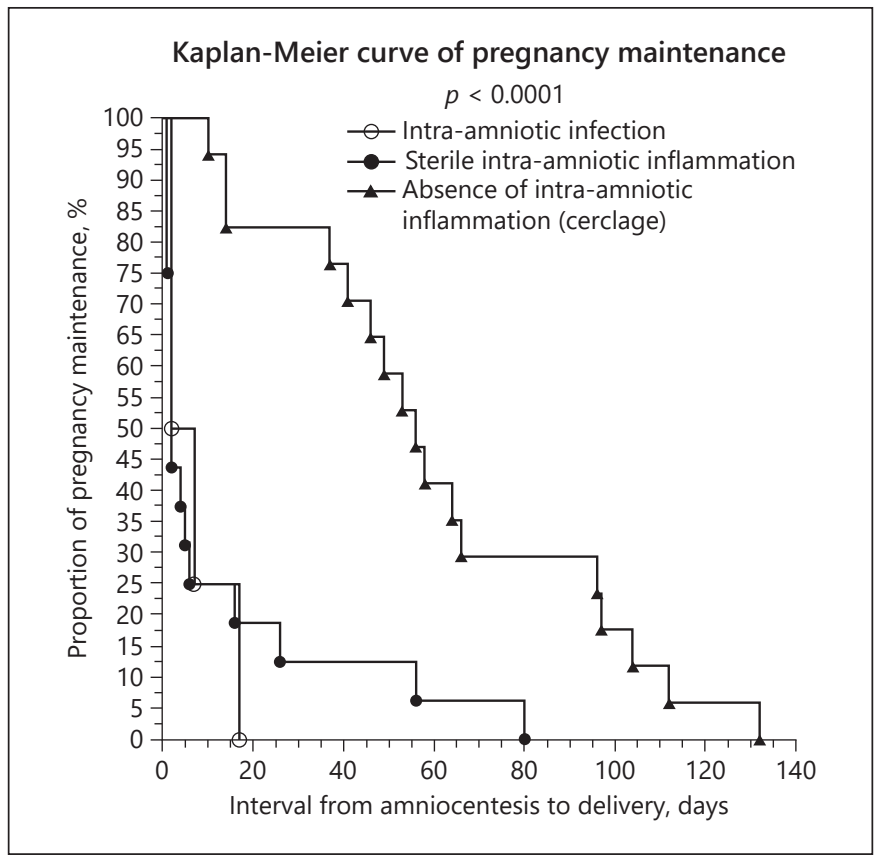

Fig. 2. Proportion of pregnancy maintenance in women with cervical insufficiency with prolapsed membranes: (i) with intra-amniotic infection; (ii) with sterile intra-amniotic inflammation; and (iii) without intra-amniotic inflammation, with respect to the interval between amniocentesis and delivery.

sterile intra-amniotic inflammation, respectively. Intraamniotic infection was caused by (i) Bacteroides ureolyticus in 2 women; (ii) Streptococcus agalactiae in 1 woman; and (iii) Candida albicans in 1 woman. IL-6 concentrations in amniotic fluid at the time of admission have been shown in Figure 1.

\section{Interval from Amniocentesis to Delivery}

The intervals from amniocentesis to delivery have been compared in Figure 2. Both, women with intraamniotic infection and those with sterile intra-amniotic inflammation, had a shorter interval from amniocentesis to delivery (intra-amniotic infection: median 5 days, IQR 2-15, $p<0.0001$; sterile intra-amniotic inflammation: median 2 days, IQR $1-14, p<0.0001$ ) than women with the absence of intra-amniotic inflammation (median 56 days, IQR 39-97). No differences in the interval from amniocentesis to delivery was found between women with intra-amniotic infection and those with sterile intra-amniotic inflammation $(p=$ $0.97)$. 


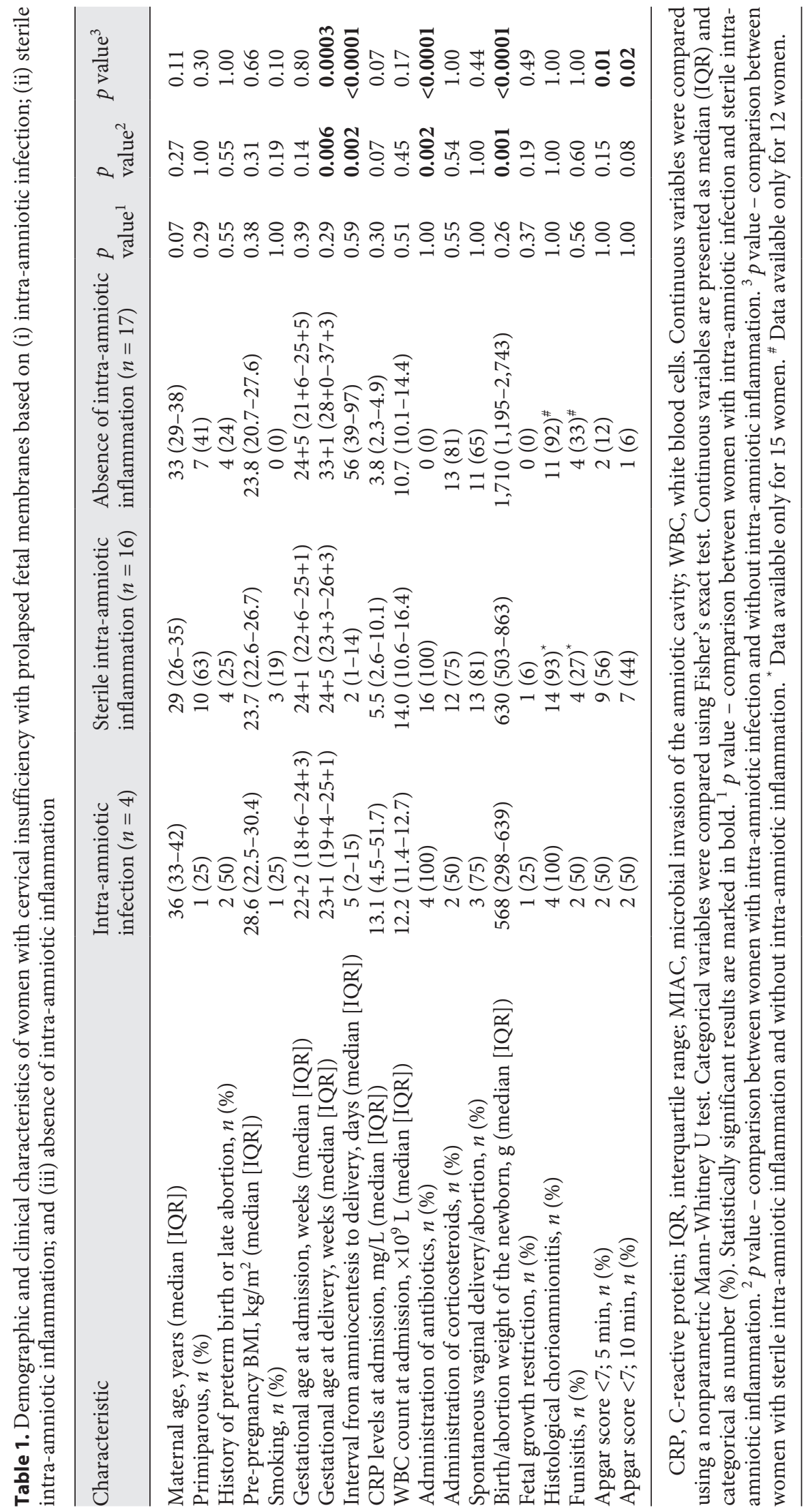




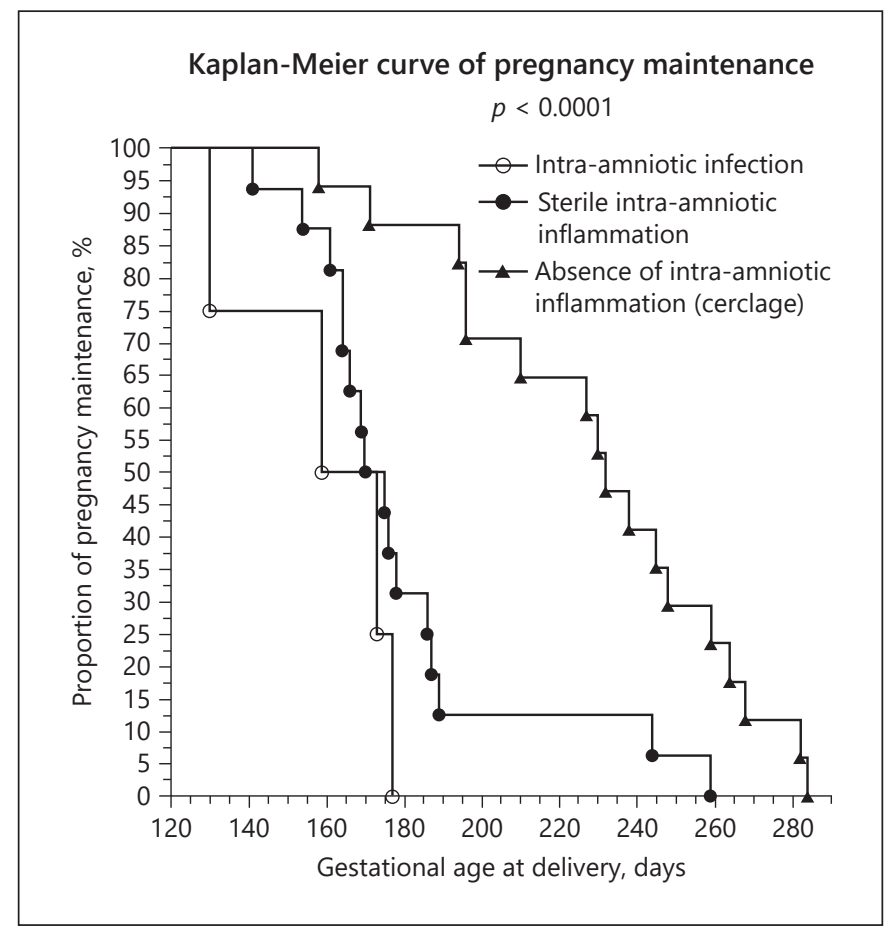

Fig. 3. Proportion of pregnancy maintenance in women with cervical insufficiency with prolapsed membranes: (i) with intra-amniotic infection; (ii) with sterile intra-amniotic inflammation; and (iii) without intra-amniotic inflammation, with respect to gestational age at delivery.

\section{Gestational Age at Delivery}

Gestational ages at delivery have been presented in Figure 3. Women with intra-amniotic infection and sterile intra-amniotic inflammation had a lower gestational age at delivery (intra-amniotic infection: median $23+1$, IQR $19+4-25+1, p<0.0001$; sterile intra-amniotic inflammation: median $24+5$, IQR $23+3-26+3$, $p=$ $0.0004)$ than women with the absence of intra-amniotic inflammation (median $33+1$, IQR $28+0-37+3$ ). No differences in gestational age at delivery was identified between women with intra-amniotic infection and those with sterile intra-amniotic inflammation $(p=0.17)$.

\section{Follow-Up Amniocentesis in Women with Intra-}

\section{Amniotic Inflammation}

In total, $25 \%(5 / 20)$ of the women with intra-amniotic inflammation had a latency longer than 7 days and underwent follow-up amniocentesis when antibiotic therapy ended. One woman had intra-amniotic infection (caused by Candida albicans) and 4 women had sterile intra-amniotic inflammation.

Cervical Insufficiency with Prolapsed

Fetal Membranes

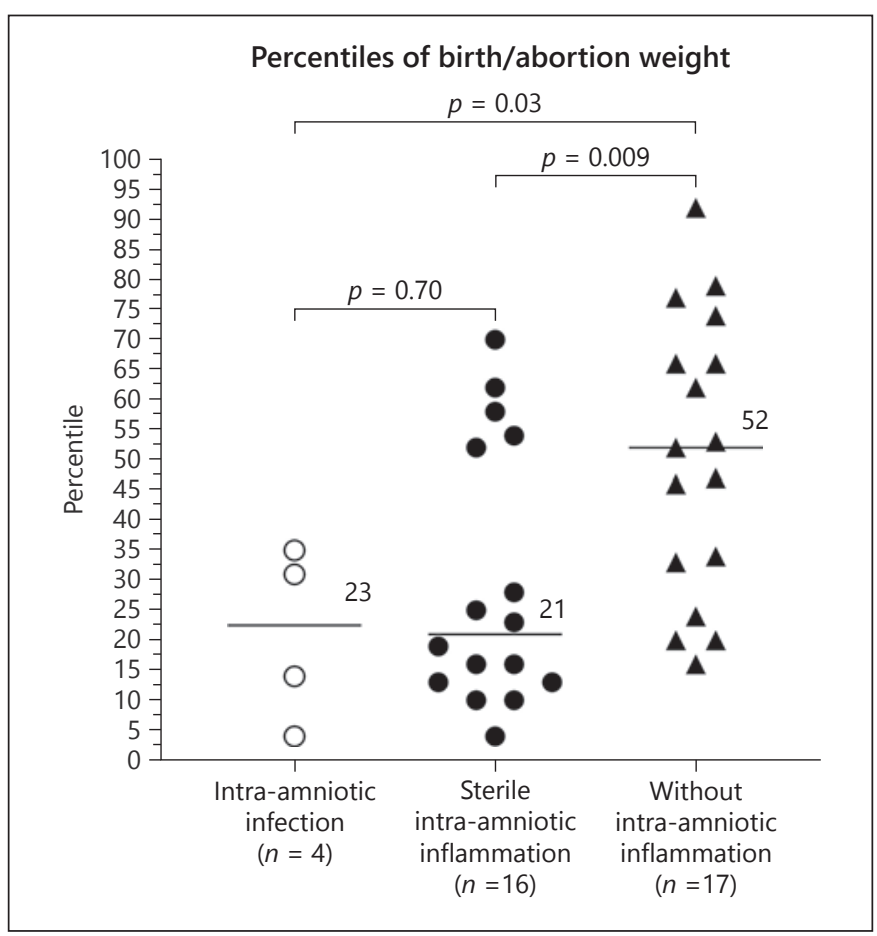

Fig. 4. Percentile distribution of birth/abortion weight of newborns from pregnancies with cervical insufficiency with prolapsed membranes: (i) with intra-amniotic infection; (ii) with sterile intra-amniotic inflammation and (iii) without intra-amniotic inflammation.

No reduction in the concentration of IL- 6 in amniotic fluid at the time of a follow-up amniocentesis was seen in the woman with intra-amniotic infection $(50,000 \mathrm{pg} / \mathrm{mL}$ in both amniocenteses). The presence of Candida albicans in amniotic fluid persisted at the time of the followup amniocentesis.

All women with sterile intra-amniotic inflammation showed a reduced concentration of IL- 6 in amniotic fluid at the time of follow-up amniocentesis. Resolution of sterile intra-amniotic inflammation was seen in 75\% (3/4) of them. One woman with persistent sterile intra-amniotic inflammation at the time of follow-up amniocentesis had a reduced concentration of IL-6 $(3,079 \mathrm{pg} / \mathrm{mL})$, but it was still above the cutoff value $(3,000 \mathrm{pg} / \mathrm{mL})$. Resolution of prolapsed membranes was observed in all women with resolution of sterile intra-amniotic inflammation.

\section{Percentiles of Birth/Abortion Weight}

There were no differences in the presence of fetal growth restriction and defined birth/aborted weight $<10$ th percentile among the subgroups (Table 1 ). Women 


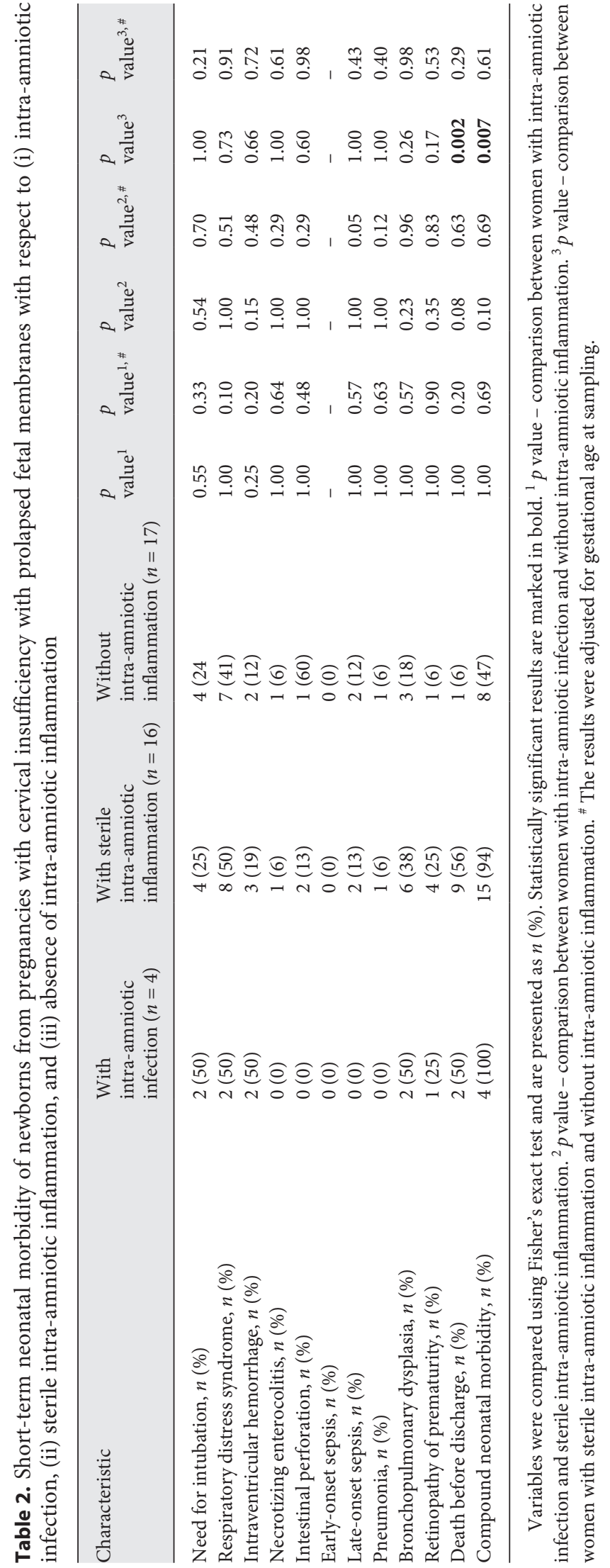

with intra-amniotic infection and sterile intra-amniotic inflammation had lower percentiles of birth/abortion weights of newborns/fetuses than women without intraamniotic inflammation as per crude analysis (intra-amniotic infection: median 23 , IQR 7-34, $p=0.03$; sterile intra-amniotic inflammation: median 21, IQR 13-54, $p=$ 0.009; without inflammation: median 52, IQR 29-70; Fig. 4), as well as after adjustment for interval between amniocentesis and delivery and administration of corticosteroids (intra-amniotic infection: $p=0.03$; sterile intra-amniotic inflammation: $p=0.05$ ). A negative correlation between percentiles of birth/abortion weight and concentrations of IL- 6 in amniotic fluid as measured by lateral flow immunoassay $(n=31)$ was identified $(\rho=$ $-0.59, p<0.0005)$.

\section{Short-Term Neonatal Outcomes}

The rates of short-term neonatal outcomes of the neonates from pregnancy with cervical insufficiency with prolapsed fetal membranes have been shown in the Table 2 . The presence of sterile intra-amniotic inflammation was associated with a higher rate of death before discharge $(p=0.002)$ and compound neonatal morbidity $(p=0.007)$ as per crude analysis, but this was not so after adjustment for gestational age at delivery.

\section{Discussion}

The main findings of this study on women with cervical insufficiency with prolapsed fetal membranes are as follows: (i) the frequency of intra-amniotic infection and sterile intra-amniotic inflammation were 11 and $43 \%$, respectively; (ii) both types of intra-amniotic inflammation were associated with a shorter interval from amniocentesis to delivery and lower gestational age at delivery; (iii) antibiotic therapy reduced the intensity of sterile intraamniotic inflammation in a fraction of the cases; (iv) intra-amniotic infection and sterile intra-amniotic inflammation were associated with a reduction of fetal growth, although it did not fulfill the criteria for fetal growth restriction; and (v) gestational age at delivery was the main determinant of short-term neonatal morbidity in women with cervical insufficiency with prolapsed fetal membranes.

\section{Intra-Amniotic Infection and Sterile Intra-Amniotic} Inflammation in Other Pregnancy Pathologies

Recent advances in microbiology that allow detection of microbial nucleic acids in amniotic fluid have provided 
a unique opportunity to identify difficult to culture and non-cultivable microorganisms in amniotic fluid. A combination of traditional culturing methods and modern molecular biology methods allows precise dissection of both phenotypes of intra-amniotic inflammation. The prevalence of intra-amniotic infection, a microorganismdriven form of intra-amniotic inflammation, has been shown to be $11 \%$ in pregnancies with spontaneous preterm labor with intact membranes [21], 21-29\% in pregnancies with preterm prelabor rupture of membranes (PPROM) $[22,34]$, and $2 \%$ in pregnancies with a short cervix [23]. The rate of sterile intra-amniotic inflammation, a non-microorganism-driven type of intra-amniotic inflammation, was shown to be higher than the rate of intra-amniotic infection in pregnancies with spontaneous preterm labor with intact membranes (26\%) [21] and with a short cervix (10\%). In PPROM pregnancies, data on the rate of sterile intra-amniotic inflammation vary between 4 and 29\% [22, 34].

\section{Intra-Amniotic Infection and Sterile Intra-Amniotic} Inflammation in Cervical Insufficiency with Prolapsed Fetal Membranes

In this study, we found that sterile intra-amniotic inflammation represents a dominant form of intra-amniotic inflammation in pregnancies with cervical insufficiency and prolapsed fetal membranes. This kind of inflammation was found in almost half of the included pregnancies and was responsible for four-fifths of all intra-amniotic inflammation cases. This observation is in line with a study by Lee et al. [14], where 73\% (38/52) of women with cervical insufficiency between the gestational age of 17-29 weeks had amniotic fluid levels of matrix metalloproteinase (MMP)- $8>23 \mathrm{ng} / \mathrm{mL}$ and negative cultures of amniotic fluid.

The prevalence of MIAC, defined either by a positive culture of amniotic fluid and/or positive PCR for Ureaplasma spp. in pregnancies with cervical insufficiency with prolapsed fetal membranes has been previously reported to range between 8 and $52 \%[10,13-16,35,36]$. However, the rate of intra-amniotic infection (defined as a positive culture along with the elevation of amniotic fluid inflammatory mediator) has been reported only by a few studies. Lee et al. [14] found intra-amniotic infection (positive culture and MMP-8 $>23 \mathrm{ng} / \mathrm{mL}$ ) in $8 \%(4 / 52)$ of the women. Diego Almela et al. [13] identified intra-amniotic infection (defined as a positive culture along with amniotic fluid IL-6 $>2.5 \mathrm{ng} / \mathrm{mL}$ ) in 13\% (4/31) of women. In this study, MIAC was assessed more thoroughly than in the previous studies using 3 complementary methods:

Cervical Insufficiency with Prolapsed

Fetal Membranes (i) aerobic and anaerobic cultivation; (ii) specific PCR for Ureaplasma spp., Mycoplasma hominis, and Chlamydia trachomatis; and (iii) nonspecific PCR for 16S rRNA gene. Using this approach, intra-amniotic infections were identified in $11 \%$ of women. This result is in line with the aforementioned studies.

There is a solid body of evidence showing that the presence of intra-amniotic inflammation in pregnancies with cervical insufficiency with prolapsed fetal membranes is associated with a shorter interval between amniocentesis and delivery $[4,12,13,17,37,38]$, lower gestational age at sampling $[4,11,13,14,17]$, and higher rates of preterm deliveries $[4,12,13,17,37]$. These findings were also confirmed by our study.

The comparison of these outcomes between 2 phenotypes of intra-amniotic inflammation (intra-amniotic infection and sterile intra-amniotic inflammation) has not been carried out before. However, results from previous studies suggest that sterile intra-amniotic inflammation might have the same deleterious effect as intra-amniotic infection [14, 17]. In 2008, Lee et al. [14] reported that women with high amniotic fluid MMP-8 concentrations, irrespective of amniotic fluid culture results, had a lower gestational age at delivery than women with low MMP-8. Four years later, Aguin et al. [17] assessed women who underwent nonelective cervical cerclage. They found that women with intra-amniotic inflammation, but with negative amniotic fluid culture, had a shorter interval between amniocentesis and delivery, lower gestational age at sampling, and higher rates of delivery $(<28$ weeks and $<32$ weeks) than those negative for both amniotic fluid culture and intra-amniotic inflammation. The findings from the present study have revealed no differences in pregnancy outcomes between women with intra-amniotic infection and those with sterile intra-amniotic inflammation.

\section{Antibiotics to Treat Intra-Amniotic Inflammation}

A recent study reported that intravenous administration of select antibiotics, either in combination [20, 39, 40] or as monotherapy [41], may reduce the intensity of intra-amniotic inflammation and even resolve both forms of intra-amniotic inflammation in women with spontaneous preterm labor [40], PPROM [39, 41], and cervical insufficiency with prolapsed fetal membranes [20]. Oh et al. [20] reported treatment success (resolution of intraamniotic inflammation or delivery $\geq 34$ weeks) in $60 \%$ of women with cervical insufficiency with intra-amniotic inflammation treated with ceftriaxone, clarithromycin, and metronidazole. Among women who experienced res- 
olution of intra-amniotic inflammation, $89 \%$ (8/9) of them showed a negative culture and PCR for Ureaplasma spp [20].

In this study, women with intra-amniotic inflammation received intravenous clarithromycin. Clarithromycin - a semi-synthetic macrolide - is not only a potent tool to treat bacterial infection, it also has other biologic properties: (i) an effective antibacterial activity against Ureaplasma spp. [42-46]; (ii) an optimal placental passage $[47,48]$; (iii) inhibition of the transcription factors of nuclear factor kappa B [49-51] and activator protein-1 $[52,53]$ (major regulators in pro-inflammatory response); and (iv) inhibition of the production of pro-inflammatory cytokines. [53-60]. Administration of intravenous clarithromycin was associated with treatment success in $15 \%(3 / 20)$ of women with intra-amniotic inflammation, particularly those with sterile intra-amniotic inflammation. Interestingly, reduction in IL-6 concentrations at the time of follow-up amniocentesis was seen only in women with sterile intra-amniotic inflammation who initially showed just mild elevation in amniotic fluid IL-6 concentrations. This suggests that, among women with cervical insufficiency with prolapsed fetal membranes, those with milder forms of sterile intra-amniotic inflammation could be positively impacted by intravenous clarithromycin. However, we are aware of the limited sample size of women in whom a follow-up amniocentesis was performed. Therefore, this observation should be noted with caution.

\section{Intra-Amniotic Inflammation and Restriction of Fetal Growth}

Restriction of fetal growth has multiple etiologies and a long preclinical phase $[61,62]$. Therefore, fetal growth restriction is considered to be one of the "great obstetrical syndromes" [61-65]. There is a plethora of evidence on the association between infection/inflammation and fetal growth: (i) the presence of histological chorioamnionitis is more frequent in growth restricted infants from preterm and term deliveries [66]; (ii) among pregnancies with small-for-gestational-age fetuses, $6 \%$ had MIAC, $2 \%$ had intra-amniotic infection, and $6 \%$ had sterile intraamniotic inflammation [67]; (iii) maternal bacterial infection may alter fetal growth $[68,69]$; (iv) concentrations of IL- 6 , tumor necrosis factor- $\alpha$, and C-reactive protein in umbilical cord blood in small-for-gestational-age infants are increased as compared to appropriate-for-gestational age infants [70]; (v) chronic hypoxia leads to elevation of IL-6 in fetal blood in animals model [71]; and (vi) chronic maternal infection/inflammation may result in small-for-gestational-age fetuses in animal models [72, 73]. Following this evidence, we assessed the differences in newborn weights, expressed as percentiles for gestational age at delivery, among newborns from pregnancies with intra-amniotic infection, with and without intraamniotic inflammation. Newborns/aborted fetuses from pregnancies with both forms of intra-amniotic inflammation had lower percentiles of birth/aborted weight than those from pregnancies without intra-amniotic inflammation. This observation is supported by a negative correlation between the amniotic fluid IL- 6 concentrations and percentiles of birth/aborted weight. Interestingly, only mild alterations of fetal growth were found in this study and they did not fulfill the criteria for fetal growth restriction (10th percentile). Given this, we cannot say whether intra-amniotic inflammation is a cause or consequence of reduction in fetal growth. This observation requires further investigation using larger cohorts of women with cervical insufficiency or different phenotypes of spontaneous preterm delivery.

In this study, pregnancies with cervical insufficiency with prolapsed fetal membranes complicated by intraamniotic infection and sterile intra-amniotic inflammation were seen to have a lower gestational age at delivery than pregnancies without intra-amniotic inflammation. Gestational age at delivery is a more important determinant of short-term neonatal outcomes than MIAC or intra-amniotic inflammation in women with spontaneous preterm delivery and PPROM $[74,75]$. Therefore, it is not surprising that, after adjustment for gestational age, we could not discern any differences in selected aspects of short-term neonatal morbidity in this study.

\section{Strengths and Limitations}

The main strength of this study was a thorough assessment of MIAC and the combination of 3 different methods, providing a unique opportunity to precisely dissect the characteristics of a subset of women with sterile intraamniotic inflammation. The second advantage is that, regardless of the relatively small sample size, the cohort was very homogenous and consists only of Caucasian women who underwent amniocentesis at the time of admission. This study also has some important limitations. First, 2 different approaches were used to assess the concentrations of IL-6 in amniotic fluid. Second, the retrospective nature of this study did not give us an opportunity to perform detailed ultrasonography assessments focused on fetal growth and uteroplacental perfusion.
Chalupska/Kacerovsky/Stranik/Gregor/ Maly/Jacobsson/Musilova 


\section{Conclusion}

In conclusion, both phenotypes of intra-amniotic inflammation, with sterile intra-amniotic inflammation being more frequent, are associated with worse outcomes in pregnancies with cervical insufficiency with prolapsed fetal membranes. Intra-amniotic infection and sterile intraamniotic inflammation are also associated with a reduction in fetal growth.

\section{Statement of Ethics}

The study was approved by the Institutional Review Board of University Hradec Kralove (July 2014: number 201408 S07P), and written informed consent was received from all participants prior to sampling. The research complies with the guidelines for human studies and was conducted ethically in accordance with the Medical Association Declaration of Helsinki.

\section{Funding Sources}

This study was supported by the project PERSONMED - Center for the Development of Personalized Medicine in Age-Related Diseases, Reg. No. CZ.02.1.01/0.0/0.0/17_048/0007441 and Faculty Hospital in Hradec Kralove (long-term organization development plan).

\section{Author Contributions}

Conceptualizations: Marian Kacerovsky and Ivana Musilova; data curation and analysis: Marian Kacerovsky, Martina Chalupska, and Jaroslav Stranik; investigation: Martina Chalupska, Marian Kacerovsky, Jaroslav Stranik, Miroslav Gregor, Jan Maly, Bo Jacobsson, and Ivana Musilova; methodology: Marian Kacerovsky and Ivana Musilova; supervision: Bo Jacobsson; writing - original draft: Marian Kacerovsky; writing - review \& editing: Martina Chalupska, Marian Kacerovsky, Jaroslav Stranik, Miroslav Gregor, Jan Maly, Bo Jacobsson, and Ivana Musilova.

\section{Conflict of Interest Statement}

The authors report that they have no conflicts of interest. The authors alone are responsible for the content and writing of the paper.

\section{References}

1 Romero R, Espinoza J, Erez O, Hassan S. The role of cervical cerclage in obstetric practice: can the patient who could benefit from this procedure be identified? Am J Obstet Gynecol. 2006;194(1):1-9.

2 American College of O, Gynecologists. ACOG practice bulletin No. 142: cerclage for the management of cervical insufficiency. Obstet Gynecol. 2014;123(2 Pt 1):372-9.

3 Roman A, Suhag A, Berghella V. Cerclage: indications and patient counseling. Clin Obstet Gynecol. 2016;59(2):264-9.

4 Monckeberg M, Valdes R, Kusanovic JP, Schepeler M, Nien JK, Pertossi E, et al. Patients with acute cervical insufficiency without intra-amniotic infection/inflammation treated with cerclage have a good prognosis. J Perinat Med. 2019;47(5):500-9.

5 Lidegaard O. Cervical incompetence and cerclage in Denmark 1980-1990. A register based epidemiological survey. Acta Obstet Gynecol Scand. 1994;73(1):35-8.

6 Robertson JE, Lisonkova S, Lee T, De Silva DA, von Dadelszen P, Synnes AR, et al. Fetal, infant and maternal outcomes among women with prolapsed membranes admitted before 29 weeks gestation. PLoS One. 2016;11(12): e0168285.

7 Daskalakis G, Papantoniou N, Mesogitis S, Antsaklis A. Management of cervical insuffi- ciency and bulging fetal membranes. Obstet Gynecol. 2006;107(2 Pt 1):221-6.

8 Naqvi M, Barth WH Jr. Emergency cerclage: outcomes, patient selection, and operative considerations. Clin Obstet Gynecol. 2016; 59(2):286-94

9 Cilingir IU, Sayin C, Sutcu H, İnan C, Erzincan S, Yener C, et al. Emergency cerclage in twins during mid gestation may have favorable outcomes: results of a retrospective cohort. J Gynecol Obstet Hum Reprod. 2018; 47(9):451-3.

10 Romero R, Gonzalez R, Sepulveda W, Brandt F, Ramirez M, Sorokin Y, et al. Infection and labor. VIII. Microbial invasion of the amniotic cavity in patients with suspected cervical incompetence: prevalence and clinical significance. Am J Obstet Gynecol. 1992;167(4 Pt 1):1086-91.

11 Mays JK, Figueroa R, Shah J, Khakoo H, Kaminsky S, Tejani N. Amniocentesis for selection before rescue cerclage. Obstet Gynecol. 2000;95(5):652-5.

12 Lee KY, Jun HA, Kim HB, Kang SW. Interleukin-6, but not relaxin, predicts outcome of rescue cerclage in women with cervical incompetence. Am J Obstet Gynecol. 2004; 191(3):784-9.

13 Diago Almela VJ, Martinez-Varea A, PeralesPuchalt A, Alonso-Diaz R, Perales A. Good prognosis of cerclage in cases of cervical insufficiency when intra-amniotic inflammation/infection is ruled out. J Matern Fetal Neonatal Med. 2015;28(13):1563-8.

14 Lee SE, Romero R, Park CW, Jun JK, Yoon $\mathrm{BH}$. The frequency and significance of intraamniotic inflammation in patients with cervical insufficiency. Am J Obstet Gynecol. 2008;198(6):633-8.

15 Bujold E, Morency AM, Rallu F, Ferland S, Tétu A, Duperron L, et al. Bacteriology of amniotic fluid in women with suspected cervical insufficiency. J Obstet Gynaecol Can. 2008; 30(10):882-7.

16 Airoldi J, Pereira L, Cotter A, Gomez R, Berghella V, Prasertcharoensuk W, et al. Amniocentesis prior to physical exam-indicated cerclage in women with midtrimester cervical dilation: results from the expectant management compared to physical exam-indicated cerclage international cohort study. Am J Perinatol. 2009;26(1):63-8.

17 Aguin E, Aguin T, Cordoba M, Aguin V, Roberts R, Albayrak S, et al. Amniotic fluid inflammation with negative culture and outcome after cervical cerclage. J Matern Fetal Neonatal Med. 2012;25(10):1990-4.

18 Charles D, Edwards WR. Infectious complications of cervical cerclage. Am J Obstet Gynecol. 1981;141(8):1065-71. 
19 Vidaeff AC, Ramin SM. Management strategies for the prevention of preterm birth: part II: update on cervical cerclage. Curr Opin Obstet Gynecol. 2009;21(6):485-90.

20 Oh KJ, Romero R, Park JY, Lee J, CondeAgudelo A, Hong J-S, et al. Evidence that antibiotic administration is effective in the treatment of a subset of patients with intra-amniotic infection/inflammation presenting with cervical insufficiency. Am J Obstet Gynecol. 2019;221(2):e1-140.

21 Romero R, Miranda J, Chaiworapongsa T, Korzeniewski SJ, Chaemsaithong P, Gotsch F, et al. Prevalence and clinical significance of sterile intra-amniotic inflammation in patients with preterm labor and intact membranes. Am J Reprod Immunol. 2014;72(5): $458-74$.

22 Romero R, Miranda J, Chaemsaithong P, Chaiworapongsa T, Kusanovic JP, Dong Z, et al. Sterile and microbial-associated intra-amniotic inflammation in preterm prelabor rupture of membranes. J Matern Fetal Neonatal Med. 2015;28(12):1394-409.

23 Romero R, Miranda J, Chaiworapongsa T, Chaemsaithong P, Gotsch F, Dong Z, et al. Sterile intra-amniotic inflammation in asymptomatic patients with a sonographic short cervix: prevalence and clinical significance. J Matern Fetal Neonatal Med. 2015; 28(11):1343-59.

24 Holst RM, Jacobsson B, Hagberg H, Wennerholm UB. Cervical length in women in preterm labor with intact membranes: relationship to intra-amniotic inflammation/microbial invasion, cervical inflammation and preterm delivery. Ultrasound Obstet Gynecol. 2006;28(6):768-74.

25 Kacerovsky M, Musilova I, Hornychova H, Kutova R, Pliskova L, Kostal M, et al. Bedside assessment of amniotic fluid interleukin-6 in preterm prelabor rupture of membranes. Am J Obstet Gynecol. 2014;211(4):385-9.

26 Musilova I, Andrys C, Holeckova M, Kolarova $\mathrm{V}$, Pliskova $\mathrm{L}$, Drahosova $\mathrm{M}$, et al. Interleukin-6 measured using the automated electrochemiluminescence immunoassay method for the identification of intra-amniotic inflammation in preterm prelabor rupture of membranes. J Matern Fetal Neonatal Med. 2018;1-131.

27 Fouhy F, Deane J, Rea MC, O’Sullivan Ó, Ross RP, O'Callaghan G, et al. The effects of freezing on faecal microbiota as determined using MiSeq sequencing and culture-based investigations. PLoS One. 2015;10(3):e0119355.

28 Chaemsaithong P, Romero R, Korzeniewski SJ, Martinez-Varea A, Dong Z, Yoon BH, et al. A rapid interleukin-6 bedside test for the identification of intra-amniotic inflammation in preterm labor with intact membranes. J Matern Fetal Neonatal Med. 2016;29(3):34959.
29 Chaemsaithong P, Romero R, Korzeniewski SJ, Martinez-Varea A, Dong Z, Yoon BH, et al. A point of care test for interleukin-6 in amniotic fluid in preterm prelabor rupture of membranes: a step toward the early treatment of acute intra-amniotic inflammation/infection. J Matern Fetal Neonatal Med. 2016;29(3):360-7.

30 Salafia CM, Weigl C, Silberman L. The prevalence and distribution of acute placental inflammation in uncomplicated term pregnancies. Obstet Gynecol. 1989;73(3 Pt 1):383-9.

31 Olsen IE, Groveman SA, Lawson ML, Clark $\mathrm{RH}$, Zemel BS. New intrauterine growth curves based on United States data. Pediatrics. 2010;125(2):e214-24.

32 Chou JH, Roumiantsev S, Singh R. PediTools electronic growth chart calculators: applications in clinical care, research, and quality improvement. J Med Internet Res. 2020;22(1):e16204.

33 Papile LA, Burstein J, Burstein R, Koffler H. Incidence and evolution of subependymal and intraventricular hemorrhage: a study of infants with birth weights less than 1,500 gm. J Pediatr. 1978;92(4):529-34.

34 Musilova I, Kutová R, Pliskova L, Stepan M, Menon R, Jacobsson B, et al. Intraamniotic inflammation in women with preterm prelabor rupture of membranes. PLoS One. 2015; 10(7):e0133929.

35 MacDougall J, Siddle N. Emergency cervical cerclage. Br J Obstet Gynaecol. 1991;98(12): 1234-8.

36 Oh KJ, Lee SE, Jung H, Kim G, Romero R, Yoon BH. Detection of Ureaplasmas by the polymerase chain reaction in the amniotic fluid of patients with cervical insufficiency. Perinat Med. 2010;38(3):261-8.

37 Hatakeyama Y, Miura H, Sato A, Onodera Y, Sato N, Shimizu D, et al. Neutrophil elastase in amniotic fluid as a predictor of preterm birth after emergent cervical cerclage. Acta Obstet Gynecol Scand. 2016;95(10):1136-42.

38 Govia RNM, Birse KD, Sepehri S, Khafipour E, Menticoglou SM, Burgener AD, et al. Amniotic fluid proteomic signatures of cervical insufficiency and their association with length of latency. Am J Reprod Immunol. 2018; 80(5):e13030.

39 Lee J, Romero R, Kim SM, Chaemsaithong P, Yoon BH. A new antibiotic regimen treats and prevents intra-amniotic inflammation/ infection in patients with preterm PROM. J Matern Fetal Neonatal Med. 2016;29(17): 2727-37.

40 Yoon BH, Romero R, Park JY, Oh KJ, Lee J, Conde-Agudelo A, et al. Antibiotic administration can eradicate intra-amniotic infection or intra-amniotic inflammation in a subset of patients with preterm labor and intact membranes. Am J Obstet Gynecol. 2019;221(2): e1-142.

41 Kacerovsky M, Romero R, Stepan M, Stranik J, Maly J, Pliskova L, et al. Antibiotic administration reduces the rate of intraamniotic inflammation in preterm prelabor rupture of the membranes. Am J Obstet Gynecol. 2020; 223(1):e1-114.
42 Bayraktar MR, Ozerol IH, Gucluer N, Celik O. Prevalence and antibiotic susceptibility of mycoplasma hominis and Ureaplasma urealyticum in pregnant women. Int J Infect Dis. 2010;14(2):e90-5.

43 Krausse R, Schubert S. In-vitro activities of tetracyclines, macrolides, fluoroquinolones and clindamycin against mycoplasma hominis and Ureaplasma ssp. isolated in Germany over 20 years. Clin Microbiol Infect. 2010; 16(11):1649-55.

44 Samra Z, Rosenberg S, Dan M. Susceptibility of Ureaplasma urealyticum to tetracycline, doxycycline, erythromycin, roxithromycin, clarithromycin, azithromycin, levofloxacin and moxifloxacin. J Chemother. 2011;23(2): 77-9.

45 De Francesco MA, Caracciolo S, Bonfanti C, Manca N. Incidence and antibiotic susceptibility of mycoplasma hominis and Ureaplasma urealyticum isolated in Brescia, Italy, over 7 years. J Infect Chemother. 2013;19(4):6217.

46 Lee MY, Kim MH, Lee WI, Kang SY, Jeon YL. Prevalence and antibiotic susceptibility of mycoplasma hominis and Ureaplasma urealyticum in pregnant women. Yonsei Med J. 2016;57(5):1271-5

47 Park HS, Ahn BJ, Jun JK. Placental transfer of clarithromycin in human pregnancies with preterm premature rupture of membranes. J Perinat Med. 2012;40(6):641-6.

48 Witt A, Sommer EM, Cichna M, Postlbauer K, Widhalm A, Gregor H, et al. Placental passage of clarithromycin surpasses other macrolide antibiotics. Am J Obstet Gynecol. 2003; 188(3):816-9.

49 Ichiyama T, Nishikawa M, Yoshitomi T, Hasegawa S, Matsubara T, Hayashi T, et al. Clarithromycin inhibits NF-kappaB activation in human peripheral blood mononuclear cells and pulmonary epithelial cells. Antimicrob Agents Chemother. 2001;45(1):44-7.

50 Wallwork B, Coman W, Mackay-Sim A, Cervin A. Effect of clarithromycin on nuclear factor-kappa B and transforming growth factorbeta in chronic rhinosinusitis. Laryngoscope. 2004;114(2):286-90.

51 Ogawa M, Suzuki J, Hishikari K, Takayama K, Tanaka H, Isobe M. Clarithromycin attenuates acute and chronic rejection via matrix metalloproteinase suppression in murine cardiac transplantation. J Am Coll Cardiol. 2008; 51(20):1977-85.

52 Abe S, Nakamura H, Inoue S, Takeda H, Saito $\mathrm{H}$, Kato $\mathrm{S}$, et al. Interleukin-8 gene repression by clarithromycin is mediated by the activator protein-1 binding site in human bronchial epithelial cells. Am J Respir Cell Mol Biol. 2000;22(1):51-60.

53 Kikuchi T, Hagiwara K, Honda Y, Gomi K, Kobayashi T, Takahashi H, et al. Clarithromycin suppresses lipopolysaccharide-induced interleukin- 8 production by human monocytes through AP-1 and NF-kappa B transcription factors. J Antimicrob Chemother. 2002;49(5):745-55. 
54 Iino Y, Toriyama M, Kudo K, Natori Y, Yuo A. Erythromycin inhibition of lipopolysaccharide-stimulated tumor necrosis factor alpha production by human monocytes in vitro. Ann Otol Rhinol Laryngol Suppl. 1992; 157:16-20.

55 Oishi K, Sonoda F, Kobayashi S, Iwagaki A, Nagatake T, Matsushima K, et al. Role of interleukin-8 (IL-8) and an inhibitory effect of erythromycin on IL-8 release in the airways of patients with chronic airway diseases. Infect Immun. 1994;62(10):4145-52.

56 Morikawa K, Oseko F, Morikawa S, Iwamoto K. Immunomodulatory effects of three macrolides, midecamycin acetate, josamycin, and clarithromycin, on human T-lymphocyte function in vitro. Antimicrob Agents Chemother. 1994;38(11):2643-7.

57 Khair OA, Devalia JL, Abdelaziz MM, Sapsford RJ, Davies RJ. Effect of erythromycin on Haemophilus influenzae endotoxin-induced release of IL- 6 , IL- 8 and sICAM- 1 by cultured human bronchial epithelial cells. Eur Respir J. 1995;8(9):1451-7.

58 Matsuoka N, Eguchi K, Kawakami A, Tsuboi M, Kawabe Y, Aoyagi T, et al. Inhibitory effect of clarithromycin on costimulatory molecule expression and cytokine production by synovial fibroblast-like cells. Clin Exp Immunol. 1996;104(3):501-8.

59 Morikawa K, Watabe H, Araake M, Morikawa S. Modulatory effect of antibiotics on cytokine production by human monocytes in vitro. Antimicrob Agents Chemother. 1996 40(6):1366-70

60 Suzuki H, Shimomura A, Ikeda K, Furukawa M, Oshima T, Takasaka T. Inhibitory effect of macrolides on interleukin-8 secretion from cultured human nasal epithelial cells. Laryngoscope. 1997;107(12 Pt 1):1661-6.
61 Romero R. The child is the father of the man. Prenat Neonat Med. 1996;1:8-11.

62 Di Renzo GC. The great obstetrical syndromes. J Matern Fetal Neonatal Med. 2009; 22(8):633-5.

63 Romero R, Espinoza J, Gotsch F, Kusanovic JP, Friel LA, Erez O, et al. The use of highdimensional biology (genomics, transcriptomics, proteomics, and metabolomics) to understand the preterm parturition syndrome. BJOG. 2006;113(Suppl 3):118-35.

64 Romero R. Prenatal medicine: the child is the father of the man. 1996. J Matern Fetal Neonatal Med. 2009;22(8):636-9.

65 Brosens I, Pijnenborg R, Vercruysse L, Romero R. The "Great obstetrical syndromes" are associated with disorders of deep placentation. Am J Obstet Gynecol. 2011;204(3):193201.

66 Williams MC, O’Brien WF, Nelson RN, Spellacy WN. Histologic chorioamnionitis is associated with fetal growth restriction in term and preterm infants. Am J Obstet Gynecol. 2000;183(5):1094-9.

67 DiGiulio DB, Gervasi MT, Romero R, Vaisbuch E, Mazaki-Tovi S, Kusanovic JP, et al. Microbial invasion of the amniotic cavity in pregnancies with small-for-gestational-age fetuses. J Perinat Med. 2010;38(5):495-502.

68 Offenbacher S, Lieff S, Boggess KA, Murtha AP, Madianos PN, Champagne CM, et al. Maternal periodontitis and prematurity. Part I: obstetric outcome of prematurity and growth restriction. Ann Periodontol. 2001;6(1):16474.

69 Eslick GD, Yan P, Xia HH, Murray H, Spurrett $B$, Talley NJ. Foetal intrauterine growth restrictions with Helicobacter pylori infection. Aliment Pharmacol Ther. 2002;16(9): 1677-82.
70 Amarilyo G, Oren A, Mimouni FB, Ochshorn Y, Deutsch V, Mandel D. Increased cord serum inflammatory markers in small-for-gestational-age neonates. J Perinatol. 2011;31(1): 30-2.

71 Yafeng D, Weijian H, Jiaxue W, Weiner CP. Chronic hypoxemia absent bacterial infection is one cause of the fetal inflammatory response syndrome (FIRS). Reprod Sci. 2009; 16(7):650-6.

72 Collins JG, Windley HW 3rd, Arnold RR, Offenbacher S. Effects of a Porphyromonas gingivalis infection on inflammatory mediator response and pregnancy outcome in hamsters. Infect Immun. 1994;62(10):4356-61.

73 Lin D, Smith MA, Champagne C, Elter J, Beck $\mathrm{J}$, Offenbacher S. Porphyromonas gingivalis infection during pregnancy increases maternal tumor necrosis factor alpha, suppresses maternal interleukin-10, and enhances fetal growth restriction and resorption in mice. Infect Immun. 2003;71(9):5156-62.

74 Rodriguez-Trujillo A, Cobo T, Vives I, Bosch J, Kacerovsky M, Posadas DE, et al. Gestational age is more important for short-term neonatal outcome than microbial invasion of the amniotic cavity or intra-amniotic inflammation in preterm prelabor rupture of membranes. Acta Obstet Gynecol Scand. 2016; 95(8):926-33.

75 Cobo T, Vives I, Rodríguez-Trujillo A, Murillo C, Ángeles MA, Bosch J, et al. Impact of microbial invasion of amniotic cavity and the type of microorganisms on short-term neonatal outcome in women with preterm labor and intact membranes. Acta Obstet Gynecol Scand. 2017;96(5):570-9. 